\title{
Event-related brain dynamics
}

\begin{abstract}
Will D. Penny, Stephan J . K Event-related potentials (ERPs) provide evidence of a direct link between cognitive events and brain electrical activity in a wide range of cognitive paradigms. It has generally been held that an ERP is the result of a set of disc rete stimulus-evoked brain events. A recent study, however, provides new evidence to suggest that some ERP components might be generated by stimulus-induced changes in ongoing brain dynamics. This is consistent with views emerging from several neuroscientific fields, suggesting that phase sync hronization of ongoing hythms across different spatio-temporal scales mediates the functional integration necessary to perform higher cognitive tasks.
\end{abstract}

The event-related potential (ERP) is a summary measure of the brain's electrical activity derived by averaging the post-stimulus electroencephalogram (EEG) over a large number of trials. Underlying this averaging process is an assumption that the ERP is generated from a set of stimulusevoked, fixed-latency, fixed-polarity brain events. In a recent article, however, Makeig et al. [1] provide evidence that some components of the ERP are generated by stimulusinduced changes in ongoing brain dynamics. This is a radically different perspective, which could cast new light onto how cognitive and perceptual processes are implemented in the brain.

Specifically, Makeig et al. were able to account for the generation, and attention-induced modulation, of a component of the visual ERP (the so-called ' $N 1$ ' - a negative 'peak' that is typically 'maximal' at $150 \mathrm{~ms}$ poststimulus), as arising from stimulusinduced 'partial phase resetting' of multiple ongoing EEG rhythms. These rhythms were identified by applying independent component analysis (ICA) to the single-trial data over a period that encompassed the $\mathrm{N} 1$ (using a window $50-250 \mathrm{~ms}$ poststimulus) and finding spatiotemporal modes that were consistent in their scalp topography and

\author{
Kiebel, J a mes M. Kilner and
frequency content across subjects. \\ These included central and posterior \\ 'al pha' rhythms, left and right 'mu' \\ rhythms, and frontal midline 'theta' \\ rhythms. Equivalent dipole modeling \\ of these components suggested they \\ originated from compact cortical \\ domains.
}

\section{Partial phase resetting}

There are several key aspects to these findings. The first relates to partial phase resetting. This refers to the phenomenon that, following each stimulus presentation, the phase of an ongoing rhythm is shifted towards a particular value in relation to the stimulus [2]. Thus, looking at the distribution of phase over many stimulus presentations, one sees a pre-stimulus distribution that is approximately uniform, changing to a post-stimulus distribution that peaks about a dominant value.

Communications theory [3] refers to this as phase-modulation (PM). The alternative view of the ERP - that it is generated by fixed-latency, fixedpolarity brain events - is generally referred to as amplitude modulation (AM) (Fig. 1).

As shown in Fig. 1, looking at the ERP alone cannot necessarily reveal whether the underlying modulation is mediated by phase or amplitude. To decide whether an AM or a PM mechanism underlies the ERP, one needs to look at the spectral characteristics of single-trial EEG. If there is no stimulus-induced increase in the power band of interest (e.g. 10 $\mathrm{Hz}$ ), then PM is the more likely mechanism. Indeed, from an inspection of data at individual electrodes, this is what Makeig et al. found. This echoes similar findings by Sayers et al. [4] who, in a study of auditory ERPS, concluded that auditory stimuli reorganize spontaneous activity in the EEG by changing the distribution of phase. Similarly, Brandt et al. [5] produced evidence to support the idea that the $\mathrm{N} 1$ component of the visual ERP is due to entrainment of ongoing alpha activity. More recently, the phenomenon of partial phase
Mick D. Rugg

resetting has been most remarkably demonstrated by J ackson et al. [6], who found that stimulation of pyramidal tract neurons in awake behaving macaque monkeys reset the phase of ongoing motor cortical beta rhythms, in both the local field potential and single-unit activity. Together these findings suggest that electrophysiological recordings are not purely amplitude-modulated, but rather, arise from an interaction between sensory input and ongoing dynamic brain activity.

\section{Neural network models}

The mechanisms of amplitude and phase modulation have been extensively studied in theoretical models of neuronal networks. These models describe how the activity producing a single trial of EEG can be generated from the underlying neural circuitry. Of the many studies worthy of mention, computational models such as that of Hoppensteadt and I zhikevich [7], for example, show how memory traces can be stored in the phase relationships between neurons oscillating at a given frequency. They suggest that the brain could use principles of radio communication, with the information transmitted via phase modulations. This study is representative of a larger body of work that uses the loosely coupled oscillator metaphor for neuronal dynamics.

In earlier landmark work on the olfactory bulb, Freeman and Schneider [8] demonstrated the existence of an AM mechanism. During inhalation of a familiar odour, the EEG is a strong, almost periodic waveform, with a spatial distribution of amplitude over the bulb that is consistently different for each specific odour. These dynamics, however, take place within an inhalationexhalation cycle where, in the exhalation stage, the EEG reverts to a chaotic 'searching' attractor. This whole cycle has been more recently modeled using a loosely coupled dynamical systems approach [9], where synchronous inhalation attractors are themselves brought 
about by phase resetting. This could explain at a cellular level why phase resetting is partial and different on each trial: to enter a synchronous attractor basin, the system must be in an appropriate region of the dynamic manifold. The trend towards a coupled, nonlinear-systems approach suggests that a mixture of AM and PM mechanisms is involved, and that neuronal transients are better understood in dynamic terms.

\section{Spatially distributed sources}

Our discussion has focused on the

modulation of activity at individual electrode sites or local cortical areas in terms of the phase resetting of an ongoing rhythm. It is generally accepted, however, that all but the very earliest components of the ERP (such as evoked components of the auditory ERP arising less than $20 \mathrm{~ms}$ post-stimulus) are likely to arise from multiple, spatially distributed sources (e.g. Ref. [10]). This issue is addressed by a second key aspect of the Makeig et al. study, in which ICA was used to find a set of spatiotemporal modes underlying the $\mathrm{N} 1$ component. These modes were shown to be in agreement with single or symmetric equivalent dipole models situated in cortical areas that were consistent across subjects. The EEG recordings were then attributed to the partial phase resetting of these multiple spatially distributed components.

Independent component analysis is useful in this two-stage approach as it leads to a parsimonious representation of the data. An alternative, spatial-decomposition approach involves combining ERP analyses with functional magnetic resonance imaging ( $\mathrm{fMRI}$ ). This has been undertaken, for example by $\mathrm{Di}$ Russo et al. [11] who, in a visual stimulation experiment, were able to locate subcomponents of the $\mathrm{N} 1$ to extrastriate cortex by registering equivalent dipoles with retinotopic maps derived from $\mathrm{fMRI}$. Spatial localization of the sources underlying the ERP is important, as it will allow for a more precise characterization of their dynamics.

\section{Multiple frequencies and neuronal transients}

In a recent review, Varela et al. [12] introduce the notion of large-scale integration, defined as communication between dynamic processes separated by $>1 \mathrm{~cm}$. The majority of work in this area considers the interaction between processes at the same frequency. An interesting aspect of the Makeig et al. study is that the $\mathrm{N} 1$ component was attributed to ongoing activity from multiple frequencies. This fits in with the more general framework of Friston, who describes a process whereby large-scale integration can arise from interactions among possibly different frequency components [13]. In an analysis of magnetoencephalogram (MEG) data, for example, Friston [14] observed a significant correlation between frontal gamma activity and parietal beta activity during self-paced hand movements.

The components identified by Makeig et al. constitute a snapshot of brain dynamics in a particular time window (50-250 ms post-stimulus). To derive this snapshot, it is necessary to assume that the dynamics are stationary during this period. More generally, however, it is thought that brain dynamics are nonstationary, and that perception is mediated by the temporary formation of dynamic ensembles that wax and wane as one moves from one cognitive state to another. This has been demonstrated in a compelling EE G study by Rodriguez \& al. [15], who showed that perception of an ambiguous figure was correlated with the formation of fronto-parietal gamma synchronization (200-350 ms). This was followed by a period of desynchronization and further synchronization over fronto-central sites, coinciding with a motor response. They suggest that this desynchronization period allows for the generation of a new dynamic ensemble, and a new cognitive state.

\section{Summary}

The emergence of a single perceptual moment relies on the functional integration of many specialized brain regions. Modern imaging methods are beginning to show the underlying correlates of such integration. The study by Makeig et al. includes a description of one such set of correlates, which has much in common with current research in event-related EEG and MEG. Moreover, the descriptions converge with new findings in animal neurophysiology and fit in with the latest research in neural-network modeling. The key contribution of the Makeig et al. paper is to link the study of event-related EEG to the study of ERPs. Indeed, Makeig et al. envisage that these two fields will merge into the study of 'event-related brain dynamics' [16] which, when combined with new computational models and signal processing methods, could soon provide a much richer picture of the brain processes underlying human cognition.

\section{Acknowledgements}

The work of W.D.P., S.J .K. and M.D.R. is supported by the Wellcome Trust.

\section{References}

1 Makeig, S. et al. (2002) Dynamic brain sources of visual evoked responses. Science 295, 690-694 2 Tass, P.A. (1999) Phase Resetting in Medicineand Biology: Stochastic Modelling and Data Analysis. Springer Verlag.

3 Roden, M.S. (1996) Analog and

Digital Communications. Prentice-Hall 4 Sayers, B.M. et al. (1974) The mechanism of auditory evoked EEG responses. Nature 247, 481-483 5 Brandt, M.E. et al. (1991) The effect of the phase of prestimulus alpha activity on the averaged visual evoked response. Electroencephalogr. Clin. Neurophysiol. 80, 241-250

$6 \mathrm{~J}$ ackson, A. et al. Rhythm generation in monkey motor cortex explored using pyramidal tract stimulation. J. Neurophysiol. (in press)

7 Hoppensteadt, F.C. and Izhikevich, E.M. (1998) Thalamo-cortical interactions modeled by weakly connected oscillators: could brain use FM radio principles? Biosystems 48 , 85-94

8 Freeman, W. and Schneider, W. (1982) Changes in spatial patterns of rabbit olfactory EEG with conditioning to odors. Psychophysiol ogy 19, 44-56 9 Breakspear, M. (2001) Perception of odors by a nonlinear model of the olfactory bulb. Int. J . Neural Syst. 11, 101-124

10 Gazzaniga, M.S. et al .

(eds) (1998) Cognitive Neuroscience: the Biol ogy of the Mind. Norton

11 Di Russo, F. et al. (2002)

Cortical sources of the early

components of the visual evoked 
potential. Hum. Brain Mapp. 15, 95111

12 Varela, F. et al. (2001)

The brainweb: phase synchronization and large-scale integration. Nat. Rev. Neurosci. 2, 229-239

13 Friston, K.J. (2000) The labile brain. I. Neuronal transients and nonlinear coupling. Philos. Trans. R.

Soc. Lond. B Biol. Sci. 355, 215-236

14 Friston, K.J . (1997)

Another neural code? Neuroimage 5,

213-220

(a) Amplitude modulation (AM)
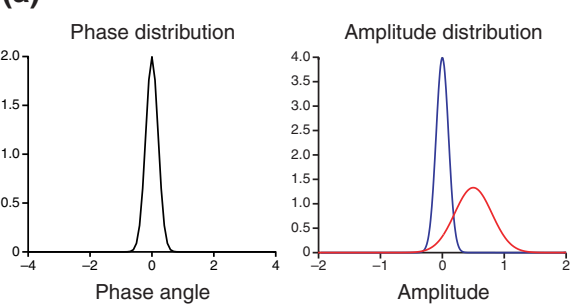

(b)

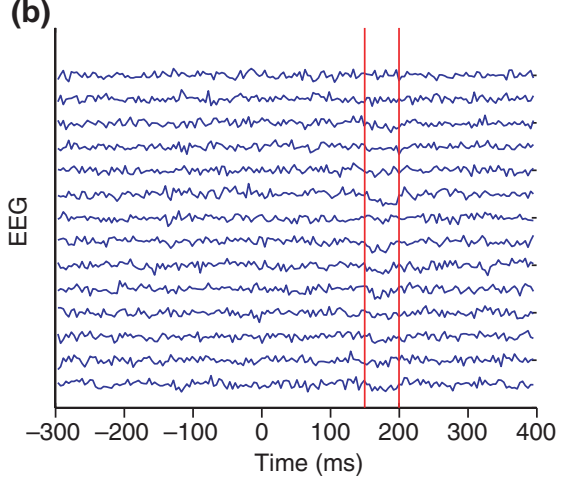

(c)

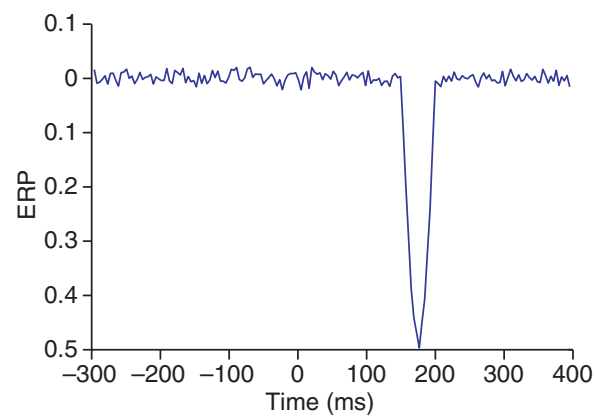

15

Rodriguez, E. et al. (1999)

Perception's shadow: long-distance

synchronization of human-brain activity. Nature 397, 430-433

16 Makeig, S. (2000) Event

related brain dynamics. In Fourth Pan-

Pacific Workshop on Brain Topography.

Irvine, CA, USA

\section{Will D. Penny}

\section{Stefan J. Kebel}

Wellc ome Department of Imaging

Neuroscience, University College

Phase modulation (PM)
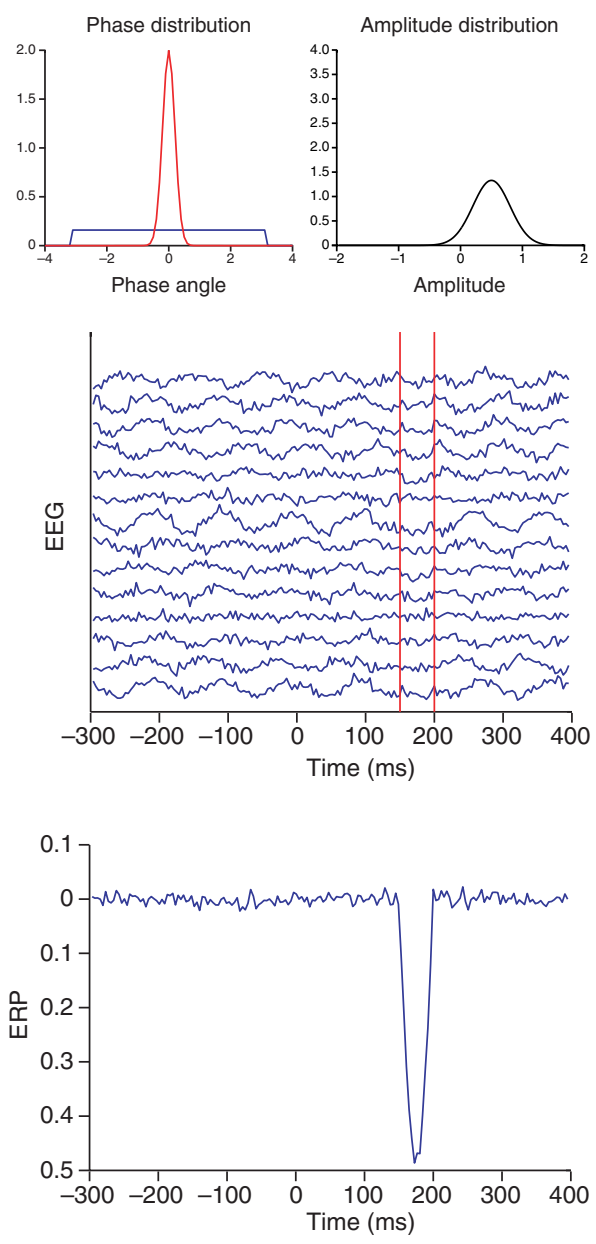

London, 12 Queen Square, London, UK WC IN 3BG.

\section{James M. Kiner}

Institut des Sciences Cognitive, UPR CNRS 9075, 67 Boulevard Pinel, 69675 BRON cedex, France.

\section{M.D. Rugg}

Institute of Cognitive Neuroscience, University College London, 17 Queen Square, London, UK WCIN 3AR.

TRENDS in Neurosciences

Fig. 1. How amplitude-modulated (AM) electroencephalogram (EEG) and phase-modulated (PM)-EEG can give rise to the same event-related potential (ERP). (a) In each trial and in each period (background or stimulus-induced), the amplitude and phase of each sinusoid a re drawn from their respective distributions. Background distributions are shown in blue and stimulus-induced distributions in red. Black indicates the same distribution for both periods. (b) Synthetic data from 15 tria ls of EEG at a single electrode. On each trial, data was generated by adding Gaussian noise onto $10-\mathrm{Hz}$ sinusoids. Within each trial there is a stimulus-induced period (150-200 ms post-stimulus, between the red lines), the rest of the trial constituting a background period. (c) The ERP computed from 1000 trials of such data. The AM and PM ERPs are identical (to within the noise limit). Note that these two examples show idealized AM and PM processes. Both mechanisms are likely to underlie real ERP data. 